\title{
Tau functions of KP solitons realized in Wiener space
}

\author{
Hidemi Aihara, Jirô Akahori, Hiroko Fujii and Yasufumi Nitta \\ Ritsumeikan University
}

July, 2011

\begin{abstract}
In this paper, a probabilistic representation of the tau functions of KP (Kadomtsev-Petviashvili) solitons in terms of stochastic areas will be presented.
\end{abstract}

\section{Introduction}

In the introduction, after giving a very short introduction to the theory of solitons following [16], we recall some existing results from probabilistic approaches.

\subsection{Solitons, tau-functions, and Sato's Grassmannian}

By solitons, we usually mean solitary wave solutions (behaving like a particle) to a class of non-linear wave equations including the KdV (Korteweg-de Vries) equation

$$
\frac{\partial u}{\partial t}=\frac{1}{4} \frac{\partial^{3} u}{\partial x^{3}}+\frac{3}{2} u \frac{\partial u}{\partial x}
$$

as the most notable example.

The first giant step in the study of solitons was made by Gardner, Greene, Kruskal and Miura [3], where they observed that (i) the eigenvalues of the Shorödinger operator

$$
\frac{\partial^{2}}{\partial x^{2}}+u(t, x)
$$


where $u$ is a solution to (1.1), is constant in time parameter $t$, and (ii) one can construct a soliton solution to (1.1) by applying the inverse scattering method, by which we mean the (mathematical) method to construct (unknown) potentials out of given scattering data, which had already been fully developed. The relation is most clearly seen when the potential is reflectionless as

$$
u(t, x)=2 \frac{d^{2}}{d x^{2}} \log \operatorname{det}(I+G(x, t))
$$

where

$$
G(x, t):=\left(\frac{\sqrt{m_{i} m_{j}} e^{\left(\eta_{i}+\eta_{j}\right) x+\left(\eta_{i}^{3}+\eta_{j}^{3}\right) t}}{\eta_{i}+\eta_{j}}\right)_{1 \leq i, j \leq n} .
$$

The constants $\eta_{j}, m_{j}, j=1, \cdots, n$ are so-called scattering data.

The observation (i) together with the awareness of the existence of the infinite invariants in [3] motivated another seminal paper by P. Lax [13], where the $\mathrm{KdV}$ equation (1.1) is understood as the compatibility between the two equations:

$$
\left\{\begin{array}{l}
\left(\frac{\partial^{2}}{\partial x^{2}}+u(t, x)\right) w(=: P w)=\kappa w, \quad(\kappa \text { is an eigenvalue }) \\
\left(\frac{\partial^{3}}{\partial x^{3}}+\frac{3}{2} u \frac{\partial}{\partial x}+\frac{3}{4} \frac{\partial u}{\partial x}\right) w(=: B w)=0 .
\end{array}\right.
$$

This compatibility is rephrased as the celebrated "Lax equation":

$$
\frac{\partial P}{\partial t}+[P, B]=0
$$

where the bracket is the commutator; $[P, B]=P B-B P$.

By considering pseudo differential operators such as $\partial^{-n}$ for $n \in \mathbf{N}$ and their infinite series, we have in fact $B=\left(P^{3 / 2}\right)_{+}$, where $(D)_{+}$is the differential operator part of the pseudo differential operator $D$. In this Lax form, the existence of the infinite many invariants can be rephrased as

$$
\frac{\partial P}{\partial x_{k}}+\left[P,\left(P^{k / 2}\right)_{+}\right]=0, \quad k=1,3,5, \cdots, 2 n+1, \cdots
$$

where $u \equiv u\left(x_{1}, x_{3}, \cdots, x_{2 n+1}, \cdots\right)$, a function of infinitely many variables. The KdV case (1.3) is retrieved by setting $x_{1}=t, x_{3}=x$. Each Lax equation generates a non-linear evolution equation with respect to $x_{2 k+1}$ since $\left[P,\left(P^{k / 2}\right)_{+}\right]$'s are all multiplication operators. The totality of the generated equations is usually called $K d V$ hierarchy. 
If we instead start with the operator

$$
L=\partial+\sum_{j=1}^{\infty} u_{j} \partial^{-j}
$$

then we still have that $\left[L,\left(L^{k}\right)_{+}\right]$are all multiplication operators, and hence we obtain infinitely many nonlinear differential equations with respect to $u_{j}$ 's of infinitely many variables $x_{1}, x_{2}, \cdots, x_{n}, \cdots$ by the Lax equations:

$$
\frac{\partial L}{\partial x_{k}}+\left[L,\left(L^{k}\right)_{+}\right]=0, \quad k=1,2, \cdots .
$$

The family is called KP hierarchy since the KP (Kadomtsev-Petviashvili) equation,

$$
\frac{3}{4} \frac{\partial^{2} u_{1}}{\partial x_{2}^{2}}=\frac{\partial}{\partial x_{1}}\left(\frac{\partial u_{1}}{\partial x_{3}}-\frac{3}{2} u_{1} \frac{\partial u_{1}}{\partial x_{1}}-\frac{1}{4} \frac{\partial^{3} u_{1}}{\partial x_{1}^{3}}\right),
$$

which is easily seen to be a generalization of the $\mathrm{KdV}$ to a two dimensional model, is deduced from the equations with $k=2$ and $k=3$. The KP hierarchy as a whole is also a generalization of the KdV hierarchy since the latter hierarchy is obtained by a reduction $\left(L^{2}\right)_{-}=0$ from the former.

The equations in $\mathrm{KP} / \mathrm{KdV}$ hierarchy are all "soliton equations" in the sense that they all have exact solutions of soliton type 1. In fact, according to Sato's theory of infinite dimensional Grassmannian ([19], see also [16, 18]), all the $u_{j}$ 's of the hierarchy are simultaneously generated from a single function called tau-function $\tau$ in the following way: determine $w_{1}, w_{2}$, etc, by

$$
\frac{\tau\left(x_{1}-\frac{1}{k}, x_{2}-\frac{1}{2 k^{2}}, \cdots\right)}{\tau\left(x_{1}, x_{2}, \cdots\right)}=1+\frac{w_{1}}{k}+\frac{w_{2}}{k^{2}}+\cdots
$$

by comparing the coefficients of $k^{-j}, j=1,2, \cdots$, and then $u_{1}, u_{2}$, etc by

$$
L=\left(1+\sum_{j=1}^{\infty} w_{j} \partial^{-j}\right) \circ \partial \circ\left(1+\sum_{j=1}^{\infty} w_{j} \partial^{-j}\right)^{-1}
$$

For example, we have

$$
u_{1}=2 \frac{\partial^{2}}{\partial x_{1}^{2}} \log \tau .
$$

\footnotetext{
${ }^{1}$ The solitons are basically rational functions of the exponential functions of the form $e^{\sum c_{i j} x_{j}}$ for some constants $c_{i j}$ 's.
} 
In particular, we see that if $\tau$ is a polynomial of $e^{\sum c_{i j} x_{j}}$ 's, then $u_{j}$ 's are all "solitons" in that they are all rational functions of $e^{\sum c_{i j} x_{j}}$ 's.

The tau functions are characterized as a solution to a family of quadratic differential equations called Hirota equations, which are nothing but Plücker relations that define Sato's infinite dimensional Grassmannian. That is to say, a tau function of the KP hierarchy is a point in the Sato's Grassmannian. It should be noted that in the Sato's theory, the KP hierarchy is the most universal one, out of which many well-known soliton equations are obtained by a reduction.

The following functions are known to be among the tau functions of the soliton solution of the KP equation:

$$
\begin{aligned}
& \tau\left(x_{1}, x_{2}, \cdots\right) \\
& =\sum_{J \subset I}\left(\prod_{i \in J} m_{i}\right)\left(\prod_{i, i^{\prime} \in J, i<i^{\prime}} \frac{\left(p_{i}-p_{i^{\prime}}\right)\left(q_{i}-q_{i^{\prime}}\right)}{\left(p_{i}-q_{i^{\prime}}\right)\left(q_{i}-p_{i^{\prime}}\right)}\right) \exp \left(\sum_{i \in J} \sum_{l=1}^{\infty}\left(p_{i}^{l}-q_{i}^{l}\right) x_{l}\right)
\end{aligned}
$$

for $I=\{1, \cdots, n\}, n \in \mathbf{N}$, where $m_{1}, \cdots, m_{n}, p_{1}, \cdots, p_{n}$, and $q_{1}, \cdots, q_{n}$ are (indefinite) constants. This is alternatively written as

$$
\tau\left(x_{1}, x_{2}, \cdots\right)=\operatorname{det}\left(I+G\left(x_{1}, x_{2}, \cdots\right)\right),
$$

where

$$
G\left(x_{1}, x_{2}, \cdots\right)=\left(\frac{\sqrt{m_{i} m_{j}}}{p_{i}-q_{j}} e^{\frac{1}{2} \sum_{l=1}^{\infty}\left\{\left(p_{i}^{l}-q_{i}^{l}\right)+\left(p_{j}^{l}-q_{j}^{l}\right)\right\} x_{l}}\right)_{i, j} .
$$

The formula (1.7) is a generalization of (1.2) since we retrieve it by (1.6) and the reductions of $q_{j}=-p_{j}, x_{l}=0$ for $l \geq 4$.

Remark 1. It should be noted that, if $f$ is a solution to a Hirota equation then so is $C e^{\sum_{j} c_{j} x_{j}} f$, for arbitrary constants $C, c_{1}, c_{2}, \cdots$. Therefore tau function is stable under the multiplication of the factor $C e^{\sum_{j} c_{j} x_{j}}$. This property will be used in the proof of Theorem 3 .

\subsection{Probabilistic approach to solitons}

As far as we know, the first attempt to represent solitons in terms of the expectation of Wiener functionals was made by S. Kotani [11] in 2000. According to 8, Kotani constructed the following correspondences. Let $\Sigma$ be the set of all pairs $\left(\sigma_{+}, \sigma_{-}\right) \equiv \sigma$ of non-negative measures each on $\mathbf{R}_{-}$such 
that $\int_{\mathbf{R}_{-}} e^{\sqrt{-1} \lambda} \sigma_{ \pm}(d \lambda)<\infty$ for any $\lambda>0$. For $\sigma \in \Sigma$, associate a Gaussian process $X^{\sigma}$ with mean 0 whose covariance $C(u, v)=\mathbf{E}[X(u) X(v)]$ is given by

$$
\begin{aligned}
C(u, v ; \sigma)= & \frac{1}{4} \int_{\mathbf{R}_{-}}(-z)^{-1 / 2}\left(e^{\sqrt{-z}(u+v)}-e^{\sqrt{-z}|u-v|}\right) \sigma_{+}(d z) \\
& +\frac{1}{4} \int_{\mathbf{R}_{-}}(-z)^{-1 / 2}\left(e^{-\sqrt{-z}|u-v|}-e^{-\sqrt{-z}(u+v)}\right) \sigma_{-}(d z) .
\end{aligned}
$$

Let $\mathcal{Q}$ be the totality of the function $q^{\sigma}$ with $\sigma \in \Sigma$, where

$$
q^{\sigma}(x)=-4 \frac{\partial^{2}}{\partial x^{2}} \log \mathbf{E}\left[\exp \left(-\frac{1}{2} \int_{0}^{x}\left|X^{\sigma}(y)\right|^{2} d s\right)\right] .
$$

Then Kotani showed that $\mathcal{Q}$ is the closure (with respect to the topology of uniform convergence on compacts) of $\cup \mathcal{Q}_{n}$, where $\mathcal{Q}_{n}$ be totality of the reflectionless potentials of scattering data consisting of $2 n$ constants.

In a somewhat different line, K. Hara and N. Ikeda [5] derived from the Fourier transform of a class of quadratic Wiener functionals a dynamics in the Grassmannian as a finite dimensional analogue to the Sato's framework (1.4)-(1.5) etc.

Soon after that N. Ikeda and S. Taniguchi [8] obtained a specific and more "stochastic analysis oriented" construction of the Gaussian process $X^{\sigma}$ in (1.8) than Kotani's method. They set

$$
X_{t}^{\sigma}=\sqrt{a}\left\langle c, \xi_{t}^{p}\right\rangle
$$

where $a>0, c \in \mathbf{R}_{+}^{n}, p \in \mathbf{R}^{n}$ and $\xi^{p}$ is an Ornstein-Uhlenbeck process in $\mathbf{R}^{n}$ starting at 0 defined as the soliton to the following SDE:

$$
d \xi_{t}=d W_{t}+\operatorname{diag}\left\{p_{1}, \cdots, p_{n}\right\} \xi_{t} d t
$$

The measure $\sigma$ in Kotani's correspondence is given as

$$
\sigma_{ \pm}(d u)=2 a^{2} \sum_{i: p_{i} \in \mathbf{R}_{ \pm}} c_{i}^{2} \delta_{-p_{i}^{2}}(d u) .
$$

Related studies and surveys concerning the quadratic Wiener functionals can be found in [20 23], and more recently in [9].

Here we remark that all the probabilistic results cited here are on $\mathrm{KdV}$ solitons, and not extendable to KP. In this paper, we will present a probabilistic representation of KP solitons using generalized stochastic areas (see Theorem 2 and Theorem 3 ). 


\subsection{Organization of the present paper}

In section 2, we will introduce Lévy's stochastic area formula and present its generalization as Theorem 2 and its proof. Then in section 3, we will show that the generalized stochastic area formula is parameterized as a tau function of KP solitons. In section 4, we will give a probabilistic interpretation of the reduction from $\mathrm{KP}$ - to $\mathrm{KdV}$-solitons. In section 5 we will present another realization, where the dimension of the Wiener space is fixed to two.

\section{A generalization of Lévy's stochastic area formula}

Let $(\Omega, \mathcal{F}, P)$ be a probability space and $W \equiv\left(W^{1}, W^{2}\right)$ be a two-dimensional Brownian motion on it. The area enclosed by the curve $s \mapsto W_{s}$ and its chord up to time $t$, which is usually called stochastic area of $W$, is given by

$$
S_{t}:=\frac{1}{2}\left(\int_{0}^{t} W_{s}^{2} d W_{s}^{1}-\int_{0}^{t} W_{s}^{1} d W_{s}^{2}\right) .
$$

The characteristic function of $S_{t}$ is explicitly given as

$$
\mathbf{E}\left[e^{\sqrt{-1} \xi S_{t}}\right]=\left(\cosh \frac{\xi t}{2}\right)^{-1} \quad(\xi \in \mathbf{R}),
$$

and conditioned one is also given explicitly as

$$
\mathbf{E}\left[e^{\sqrt{-1} \xi S_{t}} \mid W_{t}^{1}=x, W_{t}^{2}=y\right]=\frac{\xi t}{2 \sinh \frac{\xi t}{2}} e^{\frac{1}{2}\left(x^{2}+y^{2}\right)\left(1-\frac{\xi t}{2} \operatorname{coth} \frac{\xi t}{2}\right)} \quad(\xi \in \mathbf{R}),
$$

which were found by Paul Lévy [14] using Fourier series expansion of $W$. Either is often called Lévy's (stochastic area) formula(s). There have been plenty of studies related to the formulas. For example, the heat kernel of the Heisenberg group can be obtained by a slight modification of the formula ([4], see also [10]). Many alternative proofs and generalizations have been found ([24], [1], [2], [6], [7], etc).

In this paper, we give the following generalization of (2.1). In its proof, the second Lévy formula (2.2) plays a crucial role. 
Theorem 2. Let $W^{l} \equiv\left(W^{l, 1}, W^{l, 2}\right), l=1, \cdots, n$ be mutually independent two-dimensional Brownian motions starting at the origin, and stochastic areas of $W^{l}$ will be denoted by

$$
S^{l}:=\int_{0}^{1}\left(W_{s}^{l, 2} d W_{s}^{l, 1}-W_{s}^{l, 1} d W_{s}^{l, 2}\right) .
$$

Let $\Lambda:=\operatorname{diag}\left\{\lambda_{1}, \cdots, \lambda_{n}\right\}$, where $\lambda_{l}, l=1,2, \cdots, n$ are positive numbers. Let $A \equiv\left(a_{i, j}\right)_{1 \leq i, j \leq n}$ be a real $n \times n$ matrix, and $C^{ \pm}$be its symmetric and skew-symmetric part respectively, namely, $C^{ \pm}=\left(A \pm A^{*}\right) / 2$. Denote $\mathbf{W}_{t}^{i}=$ $\left(W_{t}^{1, i}, \cdots, W_{t}^{n, i}\right)$ for $i=1,2$, and define for $z \in \mathbf{C}$

$$
\begin{aligned}
\hat{S}(z) & \equiv \hat{S}_{A, \Lambda}(z) \\
& :=z \sum_{l=1}^{n} \lambda_{l} S^{l}+z\left\langle\Lambda^{\frac{1}{2}} C^{-} \Lambda^{\frac{1}{2}} \mathbf{W}_{1}^{1}, \mathbf{W}_{1}^{2}\right\rangle_{\mathbf{R}^{n}}-\frac{z^{2}}{2} \sum_{i=1,2}\left\langle\Lambda^{\frac{1}{2}} C^{+} \Lambda^{\frac{1}{2}} \mathbf{W}_{1}^{i}, \mathbf{W}_{1}^{i}\right\rangle_{\mathbf{R}^{n}} .
\end{aligned}
$$

Then, if either $\max _{l}\left|\lambda_{l}\right|$ or $\left\|C^{+}\right\|$is sufficiently small, we have

$$
\mathbf{E}\left[e^{\hat{S}(\sqrt{-1})}\right]
$$

$$
=\left|\begin{array}{cccc}
\cosh \lambda_{1}+a_{1,1} \sinh \lambda_{1} & a_{1,2} \sinh \lambda_{2} & \cdots & a_{1, n} \sinh \lambda_{n} \\
a_{2,1} \sinh \lambda_{1} & \cosh \lambda_{2}+a_{2,2} \sinh \lambda_{2} & \cdots & a_{2, n} \sinh \lambda_{n} \\
\vdots & \vdots & \ddots & \vdots \\
a_{n, 1} \sinh \lambda_{1} & a_{n, 2} \sinh \lambda_{2} & \cdots & \cosh \lambda_{n}+a_{n, n} \sinh \lambda_{n}
\end{array}\right|^{-1}
$$

Proof. We first calculate the conditional expectation of $e^{\hat{S}(\sigma)}$ conditioned by $\mathbf{W}_{1}=\left(\mathbf{W}_{1}^{1}, \mathbf{W}_{1}^{2}\right)$. By the Lévy's formula (2.2) with some analytic continuation, we have for sufficiently small $\sigma \in \mathbf{R}$ (such that the random variable $e^{\sigma \sum \lambda_{l} S^{l}}$ is integrable),

$$
\begin{aligned}
& E\left[e^{\sigma \sum_{l} \lambda_{l} S^{l}} \mid \mathbf{W}_{1}\right] \\
& =\prod_{l} \frac{\sigma \lambda_{l}}{\sin \sigma \lambda_{l}} \exp \left(-\frac{\left(W_{1}^{l, 1}\right)^{2}+\left(W_{1}^{l, 2}\right)^{2}}{2}\left(\sigma \lambda_{l} \cot \sigma \lambda_{l}-1\right)\right) .
\end{aligned}
$$

Therefore we have

$$
E\left[e^{\hat{S}(\sigma)} \mid \mathbf{W}_{1}\right]=\prod_{l} \frac{\sigma \lambda_{l}}{\sin \sigma \lambda_{l}} \exp \left(-\frac{1}{2}\left\langle(M(\sigma)-\mathbf{I}+C(\sigma)) \mathbf{W}_{1}, \mathbf{W}_{1}\right\rangle\right)
$$


where

$$
M(\sigma)=\left(\begin{array}{cc}
\sigma \Lambda \cot \sigma \Lambda & 0 \\
0 & \sigma \Lambda \cot \sigma \Lambda
\end{array}\right)
$$

with

$$
\cot \sigma \Lambda:=\operatorname{diag}\left\{\cot \sigma \lambda_{1}, \cdots, \cot \sigma \lambda_{n}\right\}
$$

as usual, and

$$
C(\sigma):=\left(\begin{array}{cc}
\sigma^{2} \Lambda^{\frac{1}{2}} C^{+} \Lambda^{\frac{1}{2}} & \sigma \Lambda^{\frac{1}{2}} C^{-} \Lambda^{\frac{1}{2}} \\
-\sigma \Lambda^{\frac{1}{2}} C^{-} \Lambda^{\frac{1}{2}} & \sigma^{2} \Lambda^{\frac{1}{2}} C^{+} \Lambda^{\frac{1}{2}}
\end{array}\right) .
$$

Since $\|M(\sigma)+C(\sigma)-I\| \rightarrow 0$ as $\sigma \rightarrow 0$, we can take $\sigma$ small enough to ensure that $M(\sigma)+C(\sigma)$ is positive definite. Then, applying quadratic Gaussian formula for such $\sigma$, we obtain

$$
\mathbf{E}\left[e^{\hat{S}(\sigma)}\right]=\prod_{l=1}^{n} \frac{\sigma \lambda_{l}}{\sin \sigma \lambda_{l}} \operatorname{det}(M(\sigma)+C(\sigma))^{-\frac{1}{2}} .
$$

We may go further as

$$
\begin{aligned}
& \operatorname{det}(M(\sigma)+C(\sigma)) \\
& =\operatorname{det}\left(\begin{array}{cc}
\sigma \Lambda^{\frac{1}{2}}\left(\Lambda^{\frac{1}{2}} \cot \sigma \Lambda+\sigma C^{+} \Lambda^{\frac{1}{2}}\right) & \sigma \Lambda^{\frac{1}{2}} C^{-} \Lambda^{\frac{1}{2}} \\
-\sigma \Lambda^{\frac{1}{2}} C^{-} \Lambda^{\frac{1}{2}} & \sigma \Lambda^{\frac{1}{2}}\left(\Lambda^{\frac{1}{2}} \cot \sigma \Lambda+\sigma C^{+} \Lambda^{\frac{1}{2}}\right)
\end{array}\right) \\
& =\operatorname{det}\left(\sigma \Lambda^{\frac{1}{2}}\left(\cot \sigma \Lambda+\sigma C^{+}+\sqrt{-1} C^{-}\right) \Lambda^{\frac{1}{2}}\right) \operatorname{det}\left(\sigma \Lambda^{\frac{1}{2}}\left(\cot \sigma \Lambda+\sigma C^{+}-\sqrt{-1} C^{-}\right) \Lambda^{\frac{1}{2}}\right)
\end{aligned}
$$

(Since $C^{-}$is skew symmetric)

$=\left\{\prod_{l}\left(\sigma \lambda_{l}\right) \operatorname{det}\left(\cot \sigma \Lambda+\sigma C^{+}+\sqrt{-1} C^{-}\right)\right\}^{2}$.

Hence (2.5) is turned into the following equality:

$$
\mathbf{E}\left[e^{\hat{S}(\sigma)}\right]=\operatorname{det}\left(\cos \sigma \Lambda+\left(\sigma C^{+}+\sqrt{-1} C^{-}\right) \sin \sigma \Lambda\right)^{-1}
$$

where $\sin \sigma \Lambda:=\operatorname{diag}\left\{\sin \sigma \lambda_{1}, \cdots, \sin \sigma \lambda_{n}\right\}$.

The right-hand-side of (2.6) is apparently meromorphic in $\sigma \in \mathbf{C}$. Now, we want to see if an analytic continuation to a domain including $z=\sqrt{-1}$ is possible or not. To see this, it suffices to check the differentiability of 
the left-hand-side of (2.6) with respect to $\sigma$. Namely, we need to check the integrability of

$$
\begin{aligned}
& \mathbf{E}\left[\frac{d}{d z} e^{\hat{S}(z)}\right] \\
& =\mathbf{E}\left[e^{\hat{S}(z)}\left(\sum_{l=1}^{n} \lambda_{l} S^{l}+\left\langle\Lambda^{\frac{1}{2}} C^{-} \Lambda^{\frac{1}{2}} \mathbf{W}_{1}^{1}, \mathbf{W}_{1}^{2}\right\rangle_{\mathbf{R}^{n}}-z \sum_{i=1,2}\left\langle\Lambda^{\frac{1}{2}} C^{+} \Lambda^{\frac{1}{2}} \mathbf{W}_{1}^{i}, \mathbf{W}_{1}^{i}\right\rangle_{\mathbf{R}^{n}}\right)\right] .
\end{aligned}
$$

Since $\hat{S}$ is quadratic Gaussian, the integrability is inherited from that of $e^{\hat{S}(z)}$ itself, which is guaranteed if either $\max _{l}\left|\lambda_{l}\right|$ or $\left\|C^{+}\right\|$is sufficiently small.

\section{Parameterization as a tau function of KP solitons}

As we have stated, a tau function $\tau$ of the $n$-soliton solution of the KadomtsevPetviashvili equation (KP equation) is expressed by

$$
\tau\left(x_{1}, x_{2}, \cdots\right)=\operatorname{det}\left(I+G\left(x_{1}, x_{2}, \cdots\right)\right),
$$

with

$$
G\left(x_{1}, x_{2}, \cdots\right)=\left(\frac{\sqrt{m_{i} m_{j}}}{p_{i}-q_{j}} e^{\frac{1}{2}\left(\xi_{i}+\xi_{j}\right)}\right)_{1 \leq i, j \leq n},
$$

where

$$
\xi_{i}=\left(p_{i}-q_{i}\right) x_{1}+\left(p_{i}^{2}-q_{i}^{2}\right) x_{2}+\cdots, \quad i=1, \cdots, n,
$$

and $m_{i}>0, p_{i}$ and $q_{i}$ are parameters.

Theorem 3. Let $P=\left(\frac{1}{p_{i}-q_{j}}\right)_{1 \leq i, j \leq n}$, and assume that $\min _{i, j}\left|p_{i}-q_{j}\right|$ is sufficiently large so that $I+P$ is invertible. Then, if we put $A=(I-P)(I+$ $P)^{-1}$ and $\Lambda:=\operatorname{diag}\left\{-\frac{1}{2}\left(\xi_{1}+\log m_{1}\right), \cdots,-\frac{1}{2}\left(\xi_{n}+\log m_{n}\right)\right\}$, we have that $\left(\mathbf{E}\left[e^{\hat{S}_{A, \Lambda}(\sqrt{-1})}\right]\right)^{-1}$, where $\hat{S}_{A, \Lambda}$ is defined by (2.3), defines a tau function of KP solitons.

Proof. Since

$$
G=e^{-\Lambda} P e^{-\Lambda}
$$


we have

$$
\begin{aligned}
\tau & =\operatorname{det}\left(I+e^{-\Lambda} P e^{-\Lambda}\right) \\
& =\operatorname{det} e^{-\Lambda} \operatorname{det}\left(e^{\Lambda}+P e^{-\Lambda}\right)=\operatorname{det}\left(I+P e^{-2 \Lambda}\right) .
\end{aligned}
$$

On the other hand,

$$
\begin{aligned}
& \operatorname{det}(\cosh \Lambda+A \sinh \Lambda) \\
& =\operatorname{det}\left(\frac{e^{\Lambda}+e^{-\Lambda}}{2}+A \frac{e^{\Lambda}-e^{-\Lambda}}{2}\right) \\
& =2^{-n} \operatorname{det}\left\{(I+A) e^{\Lambda}+(I-A) e^{-\Lambda}\right\} \\
& \left.=2^{-n} \operatorname{det}\left\{(I+A) e^{\Lambda}\right\} \operatorname{det}\left(I+(I+A)^{-1}(I-A) e^{-2 \Lambda}\right)\right) \\
& =2^{-n} \operatorname{det}(I+A) e^{-\frac{1}{2} \sum\left(\xi_{i}+\log m_{i}\right)} \operatorname{det}\left(I+P e^{-2 \Lambda}\right) .
\end{aligned}
$$

The last equality follows since

$$
A=(I-P)(I+P)^{-1} \Longleftrightarrow P=(I+A)^{-1}(I-A) .
$$

As we have stated in Remark 1, $2^{-n} \operatorname{det}(I+A) e^{-\frac{1}{2}\left(\xi_{i}+\log m_{i}\right)}$ is a trivial factor and thus by Theorem 2 we have the assertion.

\section{Reduction to Ikeda-Taniguchi's construc- tion}

As we have discussed in section 1.1, we have (1.2) by the reduction of $q_{j}=-p_{j}$ in (1.7). In this section, we review this from the perspective of stochastic analysis. We will show that when $C^{-}=0$, the expectation of the exponential of the generalized stochastic area is reduced to that of the exponential of the time integral of an Ornstein-Uhlenbeck process, which corresponds to the Taniguchi-Ikeda's construction (1.8), (1.9) and (1.10) of reflectionless potentials/tau functions of KdV solitons.

Precisely speaking, we have the following

Proposition 4. Suppose that A in Theorem 0 is symmetric. Then

$$
\mathbf{E}\left[e^{\hat{S}_{A, \Lambda}(\sqrt{-1})}\right]=\left(\mathbf{E}\left[e^{-\int_{0}^{1} X_{s}^{A, \Lambda} d s}\right]\right)^{2} e^{\operatorname{tr} \Lambda A},
$$


where $X^{A, \Lambda}=\langle(\Lambda-A \Lambda A) \xi$, $\xi\rangle$ and $\xi$ is an Ornstein-Uhlenbeck process on $\mathbf{R}^{d}$ starting at 0 and satisfying

$$
d \xi_{t}=\Lambda^{\frac{1}{2}} d B_{t}+\Lambda A \xi_{t} d t
$$

with $B$ being an $n$-dimensional standard Brownian motion.

Proof. We first note the following identity since its right-hand-side also equals to that of (2.4) with $\sigma$ replaced by $\sqrt{-1}$ (see e.g. [15]):

$$
\mathbf{E}\left[e^{\sqrt{-1} \sum_{l} \lambda_{l} S^{l}} \mid \mathbf{W}_{1}\right]=\mathbf{E}\left[e^{-\sum_{l} \frac{\lambda_{l}^{2}}{2} \int_{0}^{1}\left\{\left(W_{s}^{l, 1}\right)^{2}+\left(W_{s}^{l, 2}\right)^{2}\right\} d s} \mid \mathbf{W}_{1}\right] .
$$

Then since $C^{+}=A$ and $C^{-}=0$, we have

$$
\begin{aligned}
\mathbf{E}\left[e^{\hat{S}_{A, \Lambda}(\sqrt{-1})}\right] & =\prod_{i=1,2} \mathbf{E}\left[e^{-\sum_{l} \frac{\lambda_{l}^{2}}{2} \int_{0}^{1}\left(W_{s}^{l, i}\right)^{2} d s+\frac{1}{2}\left\langle\Lambda^{\frac{1}{2}} A \Lambda^{\frac{1}{2}} \mathbf{W}_{1}^{i}, \mathbf{W}_{1}^{i}\right\rangle}\right] \\
& =\left(\mathbf{E}\left[e^{-\sum_{l} \frac{\lambda_{l}^{2}}{2} \int_{0}^{1}\left(W_{s}^{l, 1}\right)^{2} d s+\frac{1}{2}\left\langle\Lambda^{\frac{1}{2}} A \Lambda^{\frac{1}{2}} \mathbf{W}_{1}^{1}, \mathbf{W}_{1}^{1}\right\rangle}\right]\right)^{2} .
\end{aligned}
$$

By applying Itô's formula,

$$
\begin{aligned}
& e^{-\sum_{l} \frac{\lambda_{l}^{2}}{2} \int_{0}^{1}\left(W_{s}^{l, 1}\right)^{2} d s+\frac{1}{2}\left\langle\Lambda^{\frac{1}{2}} A \Lambda^{\frac{1}{2}} \mathbf{W}_{1}^{1}, \mathbf{W}_{1}^{1}\right\rangle} \\
& =e^{\frac{1}{2} \operatorname{tr} \Lambda A} e^{\int_{0}^{1}\left\langle\Lambda^{\frac{1}{2}} A \Lambda^{\frac{1}{2}} \mathbf{W}_{s}^{1}, d \mathbf{W}_{s}^{1}\right\rangle-\frac{1}{2} \int_{0}^{1}\left|\Lambda^{\frac{1}{2}} A \Lambda^{\frac{1}{2}} \mathbf{W}_{s}^{1}\right|^{2} d s} e^{-\frac{1}{2} \int_{0}^{1}\left\langle(\Lambda-A \Lambda A) \Lambda^{\frac{1}{2}} \mathbf{W}_{s}^{1}, \Lambda^{\frac{1}{2}} \mathbf{W}_{s}^{1}\right\rangle d s} .
\end{aligned}
$$

Define $Q$ by

$$
\left.\frac{d Q}{d P}\right|_{\mathcal{F}_{1}}=e^{\int_{0}^{1}\left\langle\Lambda^{\frac{1}{2}} A \Lambda^{\frac{1}{2}} \mathbf{W}_{s}^{1}, d \mathbf{W}_{s}^{1}\right\rangle-\frac{1}{2} \int_{0}^{1}\left|\Lambda^{\frac{1}{2}} A \Lambda^{\frac{1}{2}} \mathbf{W}_{s}^{1}\right|^{2} d s} .
$$

Then by the Maruyama-Girsanov theorem, we see that $\mathbf{W}$ under $Q$ has the same law as $\xi$ of (4.1). This completes the proof.

\section{A realization in $2 \mathrm{D}-\mathrm{Wiener}$ space}

In this section, we shall show that the $2 n$-dimensional Brownian motion used to represent $n$-solitons in Theorem 2 can be replaced by a 2 -dimensional one irrespective of $n$. This observation suggests that the whole Sato's Grassmannian would be realized in 2-dimensional Wiener space. 
Let $W \equiv\left(W^{1}, W^{2}\right)$ be a 2 -dimensional Brownian motion starting at the origin, $\left\{e^{i}=\left(e_{1}^{i}, \cdots e_{n}^{i}\right), i=1, \cdots n\right\}$ be an orthonormal basis of $\mathbf{R}^{n}$, and set

$$
f_{i}(t):=\sqrt{n} \sum_{l=1}^{n} e_{l}^{i} 1_{\left[\frac{l-1}{n}, \frac{l}{n}\right)}(t), \quad i=1,2, \cdots, n .
$$

Define

$$
S_{i, j}^{+}:=\sum_{a=1,2}\left(\int_{0}^{1} f_{i}(t) d W_{t}^{a}\right)\left(\int_{0}^{1} f_{j}(t) d W_{t}^{a}\right),
$$

and

$$
S_{i, j}^{-}:=\int_{0}^{1}\left(\int_{0}^{t} f_{j}(s) d W_{s}^{2}\right) f_{i}(t) d W_{t}^{1}-\int_{0}^{1}\left(\int_{0}^{t} f_{i}(s) d W_{s}^{1}\right) f_{j}(t) d W_{t}^{2} .
$$

In this section we assume that $\lambda_{i}>0$ for all $i$. We shall denote the $(i, j)$ entry of the matrices $\Lambda^{\frac{1}{2}} C^{+} \Lambda^{\frac{1}{2}}$ and $\Lambda^{\frac{1}{2}}\left(I+C^{-}\right) \Lambda^{\frac{1}{2}}$ by $\lambda_{i, j}^{+}$, and $\lambda_{i, j}^{-}$, respectively. Note that $\lambda_{i i}^{-}=\lambda_{i}$. We assume that either $\max _{l}\left|\lambda_{l}\right|$ or $\left\|C^{+}\right\|$is sufficiently small to ensure the integrability.

Proposition 5. We have that

$$
\begin{aligned}
\mathbf{E}\left[e^{\sum_{i, j}\left(\sqrt{-1} \lambda_{i, j}^{-} S_{i, j}^{-}+\frac{1}{2} \lambda_{i, j}^{+} S_{i, j}^{+}\right)}\right] & \left.=\mathbf{E}\left[e^{\hat{S}_{A, \Lambda}(\sqrt{-1})}\right]\right) \\
& =\operatorname{det}(\cosh \Lambda+A \sinh \Lambda)^{-1} .
\end{aligned}
$$

Proof. We will show the following equivalence in law:

$$
\begin{aligned}
& \left(\sum_{i, j} \lambda_{i, j}^{-} S_{i, j}^{-}, \sum_{i, j} \lambda_{i, j}^{+} S_{i, j}^{+}\right) \\
& \stackrel{\mathrm{d}}{=}\left(\sum_{l=1}^{n} \lambda_{l} S^{l}+\left\langle\Lambda^{\frac{1}{2}} C^{-} \Lambda^{\frac{1}{2}} \mathbf{W}_{1}^{1}, \mathbf{W}_{1}^{2}\right\rangle_{\mathbf{R}^{n}}, \sum_{i=1,2}\left\langle\Lambda^{\frac{1}{2}} C^{+} \Lambda^{\frac{1}{2}} \mathbf{W}_{1}^{i}, \mathbf{W}_{1}^{i}\right\rangle_{\mathbf{R}^{n}}\right) .
\end{aligned}
$$

Let us start with the following direct calculation:

$$
S_{i, j}^{+}=n \sum_{a=1,2}\left(\sum_{k, l} e_{l}^{i} e_{k}^{j}\left(W_{k / n}^{a}-W_{(k-1) / n}^{a}\right)\left(W_{l / n}^{a}-W_{(l-1) / n}^{a}\right)\right),
$$

and

$$
\begin{gathered}
S_{i, j}^{-}=n \sum_{l=1}^{n} e_{l}^{i} e_{l}^{j} \int_{(l-1) / n}^{l / n}\left\{\left(W_{t}^{2}-W_{(l-1) / n}^{2}\right) d W_{t}^{1}-\left(W_{t}^{1}-W_{(l-1) / n}^{1}\right) d W_{t}^{2}\right\} \\
+n \sum_{k<l}\left\{e_{k}^{i} e_{l}^{j}\left(W_{k / n}^{2}-W_{(k-1) / n}^{2}\right)\left(W_{l / n}^{1}-W_{(l-1) / n}^{1}\right)\right. \\
\left.-e_{l}^{i} e_{k}^{j}\left(W_{k / n}^{1}-W_{(k-1) / n}^{1}\right)\left(W_{l / n}^{2}-W_{(l-1) / n}^{2}\right)\right\} .
\end{gathered}
$$


By the scaling property of the Brownian motion, the process

$$
\left\{\left(W_{(s+l-1) / n}^{1}-W_{(l-1) / n}^{1}, W_{(s+l-1) / n}^{2}-W_{(l-1) / n}^{2}\right): 0 \leq s \leq 1, l=1, \cdots, n\right\}
$$

is identically distributed as

$$
\left\{n^{-1 / 2}\left(W_{s}^{l, 1}, W_{s}^{l, 2}\right): 0 \leq s \leq 1, l=1, \cdots, n\right\}
$$

Here $\left\{W^{l, 1}, W^{l, 2}, l=1, \cdots, n\right\}$ are $2 n$-dimensional Brownian motions staring at the origin. In particular,

$$
\left\{\int_{(l-1) / n}^{l / n}\left\{\left(W_{t}^{2}-W_{(l-1) / n}^{2}\right) d W_{t}^{1}-\left(W_{t}^{1}-W_{(l-1) / n}^{1}\right) d W_{t}^{2},\right\}, l=1, \cdots, n\right\}
$$

is identically distributed as

$$
\left\{n^{-1} \int_{0}^{1}\left(W_{t}^{l, 2} d W_{t}^{l, 1}-W_{t}^{l, 1} d W_{t}^{l, 2}\right), l=1, \cdots, n\right\}
$$

and

$$
\left\{\left(W_{k / n}^{1}-W_{(k-1) / n}^{1}\right)\left(W_{l / n}^{2}-W_{(l-1) / n}^{2}\right), 1 \leq k, l \leq n\right\}
$$

is identically distributed as $\left\{W_{1}^{k, 1} W_{1}^{l, 2}, 1 \leq k, l \leq n\right\}$. Therefore, we have the following identity in law:

$$
\begin{aligned}
\sum_{i, j} \lambda_{i, j}^{+} S_{i, j}^{+} & \stackrel{\mathrm{d}}{=} \sum_{a=1,2} \sum_{k, l} \sum_{i, j} \lambda_{i, j}^{+} e_{l}^{i} e_{k}^{j} W_{1}^{k, a} W_{1}^{l, a} \\
& =\sum_{a=1,2}\left\langle T^{*} \Lambda^{\frac{1}{2}} C^{+} \Lambda^{\frac{1}{2}} T \mathbf{W}_{1}^{a}, \mathbf{W}_{1}^{a}\right\rangle \\
& \stackrel{\mathrm{d}}{=} \sum_{a=1,2}\left\langle\Lambda^{\frac{1}{2}} C^{+} \Lambda^{\frac{1}{2}} \mathbf{W}_{1}^{a}, \mathbf{W}_{1}^{a}\right\rangle
\end{aligned}
$$


and

$$
\begin{aligned}
\sum_{i, j} \lambda_{i, j}^{-} S_{i, j}^{-} \stackrel{\mathrm{d}}{=} & \sum_{l=1}^{n} \sum_{i, j} \lambda_{i, j}^{-} e_{l}^{i} e_{l}^{j} \int_{0}^{1}\left(W_{t}^{l, 2} d W_{t}^{l, 1}-W_{t}^{l, 1} d W_{t}^{l, 2}\right) \\
& +\sum_{k<l} \sum_{i, j} \lambda_{i, j}^{-} e_{k}^{i} e_{l}^{j} W_{1}^{k, 2} W_{1}^{l, 1}-\sum_{i, j} \lambda_{i, j}^{-} e_{l}^{i} e_{k}^{j} W_{1}^{k, 1} W_{1}^{l, 2} \\
= & \int_{0}^{1}\left\langle T^{*} \Lambda T \mathbf{W}_{t}^{2}, d \mathbf{W}_{t}^{1}\right\rangle-\int_{0}^{1}\left\langle T^{*} \Lambda T \mathbf{W}_{t}^{1}, d \mathbf{W}_{t}^{2}\right\rangle \\
& +\left\langle T^{*} \Lambda^{\frac{1}{2}} C^{-} \Lambda^{\frac{1}{2}} T \mathbf{W}_{1}^{1}, \mathbf{W}_{1}^{2}\right\rangle \\
\stackrel{\mathrm{d}}{=} & \sum_{l=1}^{n} \lambda_{l} \int_{0}^{1}\left(W_{t}^{l, 2} d W_{t}^{l, 1}-W_{t}^{l, 1} d W_{t}^{l, 2}\right) \\
& +\left\langle\Lambda^{\frac{1}{2}} C^{-} \Lambda^{\frac{1}{2}} \mathbf{W}_{1}^{1}, \mathbf{W}_{1}^{2}\right\rangle .
\end{aligned}
$$

Here $\mathbf{W}^{a}, a=1,2$ are $n$-dimensional Brownian motions, and $T:=\left[e^{1} \cdots e^{n}\right]$, which is an orthogonal matrix since $\left\{e^{1} \cdots e^{n}\right\}$ is an orthonormal basis. Note that $\Lambda^{\frac{1}{2}} C^{-} \Lambda^{\frac{1}{2}}$ is also skew-symmetric and thus $\left(\Lambda^{\frac{1}{2}} C^{-} \Lambda^{\frac{1}{2}}\right)_{i i}=0$ for $i=$ $1, \cdots, n$.

Using the equivalences (5.1) and (5.2) in law, we can establish

$$
\left.\mathbf{E}\left[e^{\sum_{i, j}\left(\sqrt{-1} \lambda_{i, j}^{-} S_{i, j}^{-}+\frac{1}{2} \lambda_{i, j}^{+} S_{i, j}^{+}\right.}\right)\right]=\operatorname{det} \Lambda \operatorname{det}\left(\cosh \Lambda+\Lambda^{\frac{1}{2}} A \Lambda^{\frac{1}{2}} \sinh \Lambda\right)^{-1}
$$

in the same manner as we have done in the proof of Theorem 2, Since $\Lambda^{\frac{1}{2}}$ and $\sinh \Lambda$ commute, we have the assertion.

\section{References}

[1] Ph. Biane and M. Yor, Variations sur une formule de Paul Lévy, Ann. Inst. H. Poincaré Probab. Statist. 23 (1987), no. 2, suppl., 359-377 (French, with English summary). MR898500 (88k:60150)

[2] B. Duplantier, Areas of planar Brownian curves, J. Phys. A 22 (1989), no. 15, 30333048. MR1007231 (91a:60209a)

[3] C. S. Gardner, J. M. Greene, M. D. Kruskal, and R. M. Miura, Method for Solving the Korteweg-deVries Equation, Phys. Rev. Lett. 19 (1967), no. 19, 1095-1097, DOI 10.1103/PhysRevLett.19.1095.

[4] B. Gaveau, Principe de moindre action, propagation de la chaleur et estimées sous elliptiques sur certains groupes nilpotents, Acta Math. 139 (1977), no. 1-2, 95-153. MR0461589 (57 \#1574) 
[5] K. Hara and N. Ikeda, Quadratic Wiener functionals and dynamics on Grassmannians, Bull. Sci. Math. 125 (2001), no. 6-7, 481-528, DOI 10.1016/S00074497(01)01097-1. Rencontre Franco-Japonaise de Probabilités (Paris, 2000). MR1869989 (2002j:60151)

[6] N. Ikeda, S. Kusuoka, and S. Manabe, Lévy's stochastic area formula and related problems, Stochastic analysis (Ithaca, NY, 1993), Proc. Sympos. Pure Math., vol. 57, Amer. Math. Soc., Providence, RI, 1995, pp. 281-305. MR1335477 (96f:60092)

[7] N. Ikeda, S. Kusuoka, and S. Manabe, Lévy's stochastic area formula for Gaussian processes, Comm. Pure Appl. Math. 47 (1994), no. 3, 329-360, DOI 10.1002/cpa.3160470306. MR1266245 (95h:60086)

[8] N. Ikeda and S. Taniguchi, Quadratic Wiener functionals, Kalman-Bucy filters, and the KdV equation, Stochastic analysis and related topics in Kyoto, Adv. Stud. Pure Math., vol. 41, Math. Soc. Japan, Tokyo, 2004, pp. 167-187. MR2083709 (2005h:60158)

[9] The Itô-Nisio theorem, quadratic Wiener functionals, and 1-solitons, Stochastic Process. Appl. 120 (2010), no. 5, 605-621, DOI 10.1016/j.spa.2010.01.009. MR2603056 (2011c:60170)

[10] N. Ikeda and S. Watanabe, Stochastic differential equations and diffusion processes, North-Holland Mathematical Library, vol. 24, North-Holland Publishing Co., Amsterdam, 1981. MR637061 (84b:60080)

[11] S. Kotani, Probabilistic approach to reflectionless potentials, Symposium on random matrices and related topics (Tsukuba University, 2000).

$[12] \_, K d V$ flow on generalized reflectionless potentials, Zh. Mat. Fiz. Anal. Geom. 4 (2008), no. 4, 490-528, 574 (English, with English and Ukrainian summaries). MR2485241 (2009m:37194)

[13] P. D. Lax, Integrals of nonlinear equations of evolution and solitary waves, Comm. Pure Appl. Math. 21 (1968), 467-490. MR0235310 (38 \#3620)

[14] P. Lévy, Wiener's random function, and other Laplacian random functions, Proceedings of the Second Berkeley Symposium on Mathematical Statistics and Probability, 1950, University of California Press, Berkeley and Los Angeles, 1951, pp. 171-187. MR0044774 (13,476b)

[15] R. Mansuy and M. Yor, Aspects of Brownian motion, Universitext, Springer-Verlag, Berlin, 2008. MR2454984 (2010a:60278)

[16] T. Miwa, M. Jimbo, and E. Date, Solitons, Cambridge Tracts in Mathematics, vol. 135, Cambridge University Press, Cambridge, 2000. Differential equations, symmetries and infinite-dimensional algebras; Translated from the 1993 Japanese original by Miles Reid. MR1736222 (2001a:37109)

[17] S. Novikov, S. V. Manakov, L. P. Pitaevskiı̌, and V. E. Zakharov, Theory of solitons, Contemporary Soviet Mathematics, Consultants Bureau [Plenum], New York, 1984. The inverse scattering method; Translated from the Russian. MR779467 (86k:35142) 
[18] M. Sato, The KP hierarchy and infinite-dimensional Grassmann manifolds, Theta functions - Bowdoin 1987, Part 1 (Brunswick, ME, 1987), Proc. Sympos. Pure Math., vol. 49, Amer. Math. Soc., Providence, RI, 1989, pp. 51-66. MR1013125 (91d:58107)

[19] M. Sato and Y. Sato, Soliton equations as dynamical systems on infinite-dimensional Grassmann manifold, Nonlinear partial differential equations in applied science (Tokyo, 1982), North-Holland Math. Stud., vol. 81, North-Holland, Amsterdam, 1983, pp. 259-271. MR730247 (86m:58072)

[20] S. Taniguchi, Lévy's stochastic area and the principle of stationary phase, J. Funct. Anal. 172 (2000), no. 1, 165-176, DOI 10.1006/jfan.1999.3540. MR1749870 (2001g:60135)

[21] - On Wiener functionals of order 2 associated with soliton solutions of the KdV equation, J. Funct. Anal. 216 (2004), no. 1, 212-229, DOI 10.1016/j.jfa.2003.11.002. MR2091362 (2005k:60172)

[22] _ Brownian sheet and reflectionless potentials, Stochastic Process. Appl. 116 (2006), no. 2, 293-309, DOI 10.1016/j.spa.2005.09.005. MR2197979 (2007e:60064)

[23] _ Stochastic analysis and the KdV equation, Stochastic analysis and partial differential equations, Contemp. Math., vol. 429, Amer. Math. Soc., Providence, RI, 2007, pp. 245-256. MR2391539 (2009m:37211)

[24] M. Yor, Remarques sur une formule de Paul Lévy, Seminar on Probability, XIV (Paris, 1978/1979), Lecture Notes in Math., vol. 784, Springer, Berlin, 1980, pp. 343-346 (French). MR580140 (82c:60144) 